\title{
Análisis térmico e hidráulico de diferentes geometrías de tubos para mejorar el desempeño de un radiador de automóvil
}

\section{Thermal and hydraulic analysis of different tube geometries to improve the performance of an automotive radiator}

\author{
ZUÑIGA-CERROBLANCO, José Luis †ै*’, HORTELANO-CAPETILLO, Juan Gregorio’, COLLAZO- \\ BARRIENTOS, Juan Carlos"“ y HERNANDEZ-GUERRERO, Abel"”
}

Universidad Politécnica de Juventino Rosas. Departamento de Ingeniería en Sistemas Automotrices. Calle Hidalgo 102, Comunidad de Valencia, Santa Cruz de Juventino Rosas, Guanajuato, México.

"Universidad Politécnica de Aguascalientes. Departamento de Ingeniería Mecánica Automotriz.

"'Universidad de Guanajuato. Departamento de Ingeniería Mecánica. México.

ID $1^{\text {er }}$ Autor: José Luis, Zuñiga-Cerroblanco / ORC ID: 0000-0003-0493-8197, CVU CONACYT ID: 208410

ID $1^{\mathrm{er}}$ Coautor: Juan Gregorio, Hortelano-Capetillo / ORC ID: 0000-0002-3702-4853, CVU CONACYT ID: 347496

ID $2^{\text {do }}$ Coautor: Juan Carlos, Collazo-Barrientos / ORC ID: 0000-0002-7436-4523, CVU CONACYT ID: 208634

ID $3^{\text {er }}$ Coautor: Abel, Hernandez-Guerrero / SCOPUS Author ID: 55989696500, SNI CONACYT ID: 12116

DOI: $10.35429 /$ JIE.2020.11.4.13.23

Recibido Enero 15, 2020; Aceptado Marzo 31, 2020

\begin{abstract}
Resumen
La industria automotriz día con día requiere motores más potentes y compactos, esto tiene como consecuencia que los sistemas de enfriamiento deban mejorar y utilizar nuevas tecnologías para cumplir con en el objetivo de mantener a una temperatura optima el motor, estos sistemas deben ajustarse a dimensiones y peso establecidos para no aumentar el gasto de combustible. Es por ello que en el presente trabajo se analiza numéricamente el desempeño térmico e hidráulico de un radiador de automóvil. La investigación se centra en analizar diferentes geometrías para los tubos que componen el radiador, dentro de los cuales se utiliza una mezcla de agua $80 \%$ y etilenglicol $20 \%$ como fluido de enfriamiento. En los resultados se reportan los números de Nusselt global para las diferentes geometrías, así como también la caída de presión total a lo largo del tubo del radiador. Se realiza una comparación del desempeño térmico e hidráulico para las diferentes geometrías analizadas, también se calcula la generación de entropía para cada geometría. Los resultados permiten elegir la mejor geometría para aumentar la transferencia de calor, así como también, elegir la que presenta un mejor balance entre la generación de entropía debido a la transferencia de calor y a la caída de presión.
\end{abstract}

Radiador, Transferencia de calor, Caída de presión

\begin{abstract}
Nowadays the automotive industry requires more powerful and compact engines, which demand that the cooling systems must be improved using new technologies to attend the aim to maintain the engine working at optimum temperature, the cooling system must be adjusted to the dimensions and weight set to avoid the increase of fuel expense. In the present work a numerical study to analyze the thermal and hydraulic performance of a car radiator is carried out. The research focuses on analyzing different geometries for the tubes that make up the radiator, inside of tubes a mixture of $80 \%$ water and $20 \%$ ethylene glycol is used as the cooling fluid. On the results the global Nusselt numbers for the different geometries, as well as the total pressure drop along the radiator tube are reported. A comparison of the thermal and hydraulic performance for the different geometries analyzed is made. From the results the best geometry to increase heat transfer is chosen, as well as the geometry with the best balance between entropy generation due to heat transfer and pressure drop is chosen.
\end{abstract}

Citación: ZUÑIGA-CERROBLANCO, José Luis, HORTELANO-CAPETILLO, Juan Gregorio, COLLAZOBARRIENTOS, Juan Carlos y HERNANDEZ-GUERRERO, Abel. Análisis térmico e hidráulico de diferentes geometrías de tubos para mejorar el desempeño de un radiador de automóvil. Revista de Ingeniería Industrial. 2020. 4-11:13-23.

*Correspondencia al Autor (Correo Electrónico:jzunigac_ptc@upjr.edu.mx)

$\dagger$ Investigador contribuyendo como primer autor. 


\section{Introducción}

A lo largo de los años, la industria automotriz se ha enfrentado a diferentes retos, uno de los cuales es que los motores tengan mayor potencia, debido a esto se desarrollaron motores potentes, pero este desarrollo trajo como consecuencia que el gasto de combustibles de dichos motores aumentara considerablemente y debido al alza en el precio de los combustibles, el mantenimiento de automóviles con este tipo de motores es relativamente costoso comparado con motores menos potentes; otro de los problemas es el calentamiento del motor, motores con una mayor potencia requieren de un mejor sistema de enfriamiento. Es por ello, que se deben desarrollar diferentes técnicas y tecnologías para que un motor con una mayor potencia sea ahorrador de combustible y se mantenga a una temperatura de operación adecuada.

Una de las opciones para el ahorro de combustible es que el automóvil sea más ligero, esto se logra reduciendo el tamaño de los componentes principales del automóvil, de esta forma el automóvil pesará menos y como consecuencia directa se tendrá un ahorro de combustible.

Existen diferentes sistemas en el automóvil, los cuales pueden ayudar a ahorrar combustible si estos trabajan de forma eficiente, uno de los sistemas importantes es el del enfriamiento del motor, el cual consiste principalmente en un radiador, bomba para la circulación del fluido de enfriamiento, ventilador, un intercambiador de calor para el aire del habitáculo, termostato y sensor de temperatura.

Se puede estimar que un sistema de enfriamiento para un vehículo se puede reducir en un porcentaje hasta de un $10 \%$, un ejemplo claro son los sistemas de enfriamiento que utilizan los vehículos híbridos, los cuales cuentan con un motor a combustión interna y un motor eléctrico, por lo tanto requieren de un mayor espacio en el cofre, reduciendo así el espacio para el resto de los componentes, como son los múltiples sistemas de enfriamiento para el motor a combustión, el motor eléctrico y las baterías, esto incrementa la importancia de un nuevo diseño de radiador que pueda remplazar a los múltiples sistemas de enfriamiento.
Es por lo anterior, que en este trabajo se analiza el desempeño térmico e hidráulico de un radiador de automóvil, para encontrar alternativas en el diseño de estos radiadores.

\section{Antecedentes}

Un radiador de automóvil es básicamente un intercambiador de calor compacto por el cual circula un fluido de enfriamiento que está removiendo el calor producido por la combustión en el motor, en el área de intercambiadores de calor compactos se cuenta con diferentes estudios como los publicados por (Kays \& London, 1984), quienes presentan un amplio estudio de la transferencia de calor para una gran variedad de geometrías de intercambiadores de calor compactos, así mismo (Shah \& London, 2014) desarrollan diferentes trabajos en los cuales reportan datos experimentales y analíticos para tubos planos usados en un intercambiador de calor, los cuales son utilizados regularmente en los radiadores de los automóviles.

En el área automotriz, específicamente en el área de enfriamiento de los motores, la técnica de enfriamiento usada es la misma desde hace varias décadas; dicha técnica consiste en utilizar un radiador tipo panal, el cual está compuesto de tubos en los cuales circula el fluido de enfriamiento, aletas, las cuales están soldadas al tubo y utiliza un ventilador para generar el fenómeno de transferencia de calor conocido como convección forzada sobre las aletas y los tubos, de esta forma se realiza el enfriamiento del motor, removiendo el calor proveniente de la combustión y arrojándolo al ambiente por medio del radiador. Esta técnica mencionada, la cual se basa en la transferencia de calor por convección forzada, desafortunadamente está alcanzando sus límites, ya que las demandas de remoción de calor cada vez son mayores y los fluidos enfriadores tradicionales como agua o etilenglicol, tienen propiedades termofísicas pobres para incrementar la transferencia de calor.

Lo anterior hace evidente la necesidad de trabajar en nuevos conceptos y tecnologías para mejorar los sistemas de enfriamiento del vehículo. 
Dentro de los trabajos publicados en el área de enfriamiento automotriz por medio de radiadores se encuentran los relacionados a la geometría del radiador y al fluido de enfriamiento utilizado, el cual puede ser agua o etilenglicol a diferentes concentraciones $\mathrm{o}$ incluso se puede incorporar algún tipo de nanofluido, el uso del agua puede presentar corrosión y provocará daños severos al sistema de enfriamiento. (Dittus \& Boelter, 1985) son de los primeros en desarrollar estudios experimentales de transferencia de calor para radiadores de automóvil tipo tubular, en su trabajo reportan correlaciones obtenidas para flujo laminar y turbulento utilizando agua pura como fluido de trabajo, así mismo también analizan diferentes configuraciones de los tubos, analizan los efectos de tener una o varias filas de tubos en el radiador.

Otro de los trabajos experimentales con los que se cuenta es con el publicado por (Achaichia \& Cowell, 1988) quienes estudian la transferencia de calor y la caída de presión para tubos planos y aletas de un intercambiador de calor. Desarrollan correlaciones para el numero de Stanton y factor de fricción para diferentes números de Reynolds. Por otro lado, (Farrell, Wert, \& Webb, 1991) realizan experimentos, donde examinan tubos rectangulares para radiador, analizan tubos rugosos y lisos, concluyen que los tubos que tiene una rugosidad menor presentan una transferencia de calor aceptable y una menor caída de presión.

(Dong, Chen, Zhang, \& Hu, 2010), trabajaron en una combinación novedosa para los tubos del radiador, los cuales consistían en no ser rectos, sino que presentan una forma ondulada, lo cual contribuye directamente a aumentar la transferencia de calor. En sus resultados experimentales reportan que el aumento de transferencia de calor y de la caída de presión es de alrededor de $38.7 \%$. Es decir, se tiene un impacto alto en la transferencia de calor, pero por otro lado también se afecta la caída de presión, por lo cual se requiere mayor energía para mover el fluido.
Un estudio en donde se evalúa experimentalmente el desempeño térmico de un radiador, calculando el coeficiente global de transferencia de calor de acuerdo con la técnica de $\varepsilon$-NTU es publicado por (Peyghambarzadeh, Hashemabadi, Naraki, \& Vermahmoudi, 2013), quienes usan agua y etilenglicol como fluido de enfriamiento, además agregan partículas metálicas a estos fluidos para generar un nanofluido a diferentes concentraciones $(0.15$, 0.4 y 0.65 vol. \%). Obtiene que los nanofluidos ofrecen una mejor transferencia de calor.

En el trabajo realizado por (Agudelo Ortiz, 2020), se realiza un análisis experimental para obtener un mejor rendimiento del sistema de refrigeración de un motor, por otro lado, (Avila Navas, 2020) construye un banco de pruebas para la simulación de fallas del sistema de control de refrigeración.

En cuanto a trabajos numéricos realizados en área de radiadores se tiene el publicado por (Park \& Pak, 2002), en su trabajo analizaron el fenómeno de convección en un tubo plano con forma de $\mathrm{U}$, donde los tubos tienen la característica de que contienen pequeñas ranuras que contribuyen a mejorar la transferencia de calor. En sus resultados reportan los números de Nusselt y la caída de presión a través del tubo, analizan los efectos que se tienen cuando se varían las condiciones de entrada del fluido de trabajo, así como también las condiciones del aire externo. Sus resultados contribuyen a un mejor diseño de los tubos a través de los cuales pasa el fluido de trabajo. (Huang, Mao, \& Zhao, 2011) analizan un modelo numérico en $3 \mathrm{~d}$ de las aletas que se posicionan entre los tubos en un radiador de calor. Reportan contornos de velocidad y presión, donde muestran que las aletas juegan un rol importante para mejorar la transferencia de calor en el radiador de calor.

Una geometría diferente a la del tubo plano es la analizada por (Patel, Subhedar, \& Ramani, 2017) quienes analizan numéricamente una forma oval para el tubo del radiador de automóvil y realizan una optimización para ese tipo de geometría, obteniendo los parámetros óptimos para la forma oval, la optimización se realiza en función de obtener una mayor transferencia de calor y una mínima caída de presión. 
(Kayastha, 2015), realizan un análisis numérico de un radiador de calor usando tubos elípticos, obtienen las temperaturas de salida y la caída de presión a través de ellos, para etilenglicol, diferentes espacios entre tubos y diferentes flujos másicos. Obtienen el mejor espacio entre los tubos y el mejor flujo masico que ocasiona mejor caída de presión y una máxima temperatura de salida.

(Huminic \& Huminic, 2013) hacen un análisis numérico de la transferencia de calor de diferentes configuraciones de tubos usando etilenglicol y nanofluidos. Analizan tubos circulares, ovalados y planos. Encuentran que el tubo plano ofrece una mejor transferencia de calor. Parten de un círculo y los van alargando hasta obtener un tubo plano. Otro trabajo es el desarrollado por (Delavari \& Hashemabadi, 2014), quienes investigan numéricamente la mejora en la transferencia de calor para flujo laminar y turbulento de un radiador de automóvil de tubos planos, usan como fluido de enfriamiento un nanofluido, tomando como fluido base agua y etilenglicol. Comparan los Números de Nusselt obtenidos con datos experimentales para agua y etilenglicol.

\section{Modelo Geométrico}

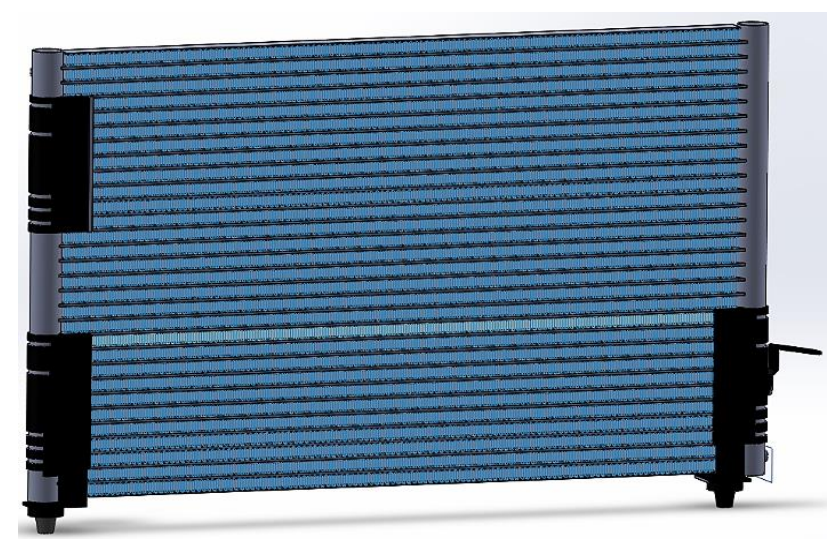

Figura 1 Configuración del radiador de automóvil Fuente: elaboración propia [SolidWorks]

Para la elaboración de la geometría del radiador se toma como base un radiador convencional, el cual está basado en la configuración de panal como puede verse en la Figura 1.
En la Figura 2 se muestra una ilustración de la configuración del radiador usada para el análisis, la cual está compuesta por tubos paralelos, los cuales son separados por aletas, dentro de los tubos fluye el fluido refrigerante, el cual puede ser agua, etilenglicol, o una mezcla de estos, para este estudio se utiliza una mezcla de $80 \%$ agua y $20 \%$ etilenglicol. Sobre la superficie de los tubos planos y las aletas pasa aire, lo cual provoca el fenómeno de convección forzada y ayuda a la transferencia de calor.

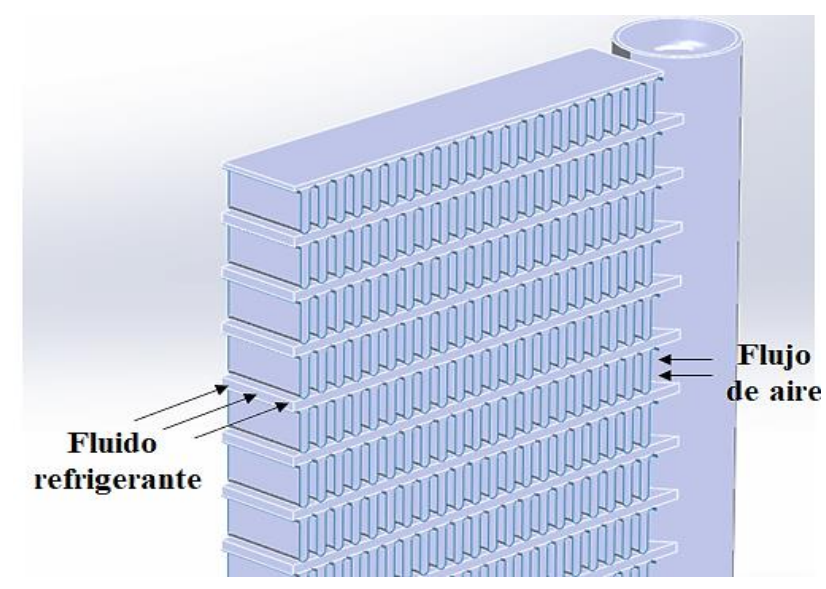

Figura 2 Geometría del radiador a analizar, tubos planos con aletas, fluido refrigerante circula en los tubos planos y flujo de aire pasa sobre la superficie de los tubos y las aletas

Fuente: elaboración propia [SolidWorks]

El análisis de este tipo de radiadores se puede simplificar al análisis de un solo tubo, sobre el cual se deben aplicar las condiciones necesarias para lograr simular su funcionamiento real en el radiador.

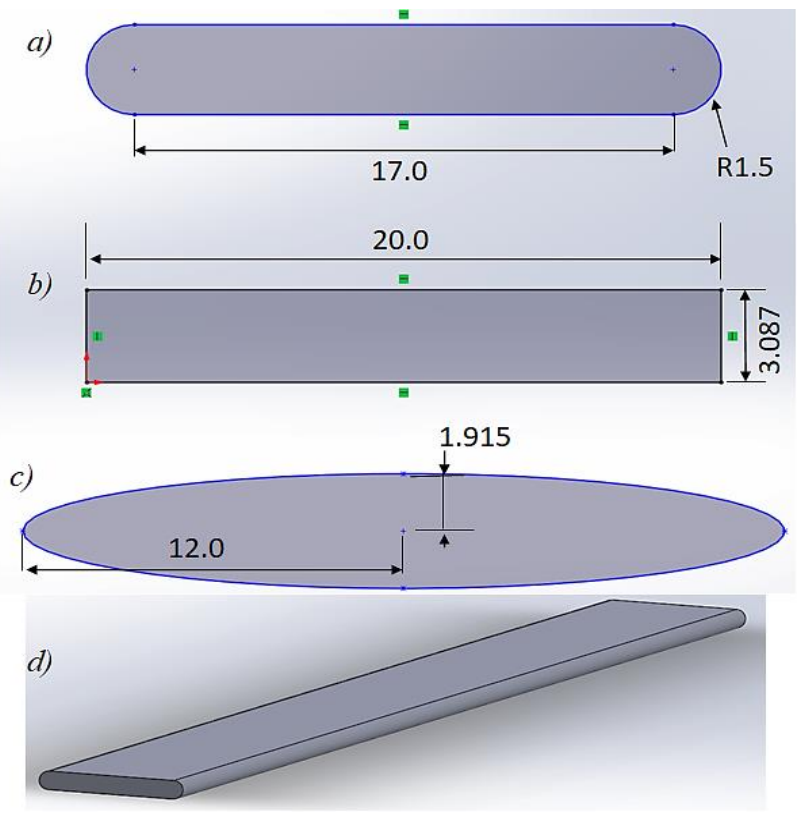

Figura 3 Geometría de los tubos del radiador que se analizan, dimensiones en $\mathrm{mm}$. a) tubo plano, b) tubo cuadrado, c) tubo elíptico y d) longitud del tubo Fuente: elaboración propia [SolidWorks]

ZUÑIGA-CERROBLANCO, José Luis, HORTELANO-CAPETILLO, Juan Gregorio, COLLAZO-BARRIENTOS, Juan Carlos y HERNANDEZ-GUERRERO, Abel. Análisis térmico e hidráulico de diferentes geometrías de tubos para mejorar el desempeño de un radiador de automóvil. Revista de Ingeniería Industrial. 2020 
El presente trabajo se basa en analizar las geometrías que pueden ser usadas en los tubos del radiador, los aspectos básicos que se deben cuidar en la elección de la geometría del tubo del radiador son, que exista una buena transferencia de calor, una menor caída de presión y que su fabricación sea de bajo costo.

Las geometrías de la sección transversal del tubo que se investigan en el presente trabajo se muestran en la Figura 3, estas son construidas en SolidWorks; a) presenta la sección transversal de un tubo plano, b) un tubo rectangular y c) un tubo con una sección transversal elíptica. Se observan las dimensiones usadas para la construcción de cada una de la geometría y d) muestra el largo del tubo tomado para el análisis, el cual fue de $31 \mathrm{~cm}$.

Las dimensiones de cada una de las geometrías presentadas en el Figura 3, se obtienen tomando el mismo diámetro hidráulico $\left(\mathrm{D}_{\mathrm{h}}=5.349 \mathrm{~mm}\right.$.), se toma el mismo diámetro hidráulico para poder hacer una comparación entre las diferentes secciones transversales. En el trabajo desarrollado por (Huminic \& Huminic, 2013) comparan diferentes secciones transversales, pero cada una de ellas tiene diferentes diámetro hidráulico.

\section{Modelo Computacional}

La geometría de los tubos es construida en SolidWorks se guarda en formato .IGES y es exportada al software ICEM-ANSYS para hacer el proceso del mallado, posteriormente se procesa y postprocesa en FLUENT para obtener los resultados numéricos.

\section{Análisis de Malla}

Se realiza discretización del dominio, iniciando con una malla que contenga poca cantidad de elemento, posteriormente se incrementa el número de elemento hasta obtener una malla con la calidad suficiente para que las variables de interés no sufran variaciones debido al número de elementos de la malla.

El Gráfico 1 muestra el análisis de malla realizado, en la gráfica tenemos la temperatura tomada a lo largo de una línea en la sección transversal en la salida del tubo.
En el Gráfico 2 se muestra a detalle la temperatura para cada una de las mallas construidas. Se puede observar que para las primeras mallas la temperatura sufre pequeños cambios, y se mantiene constante a partir de la Malla VI, por lo cual se toma esta malla como la indicada para realizar el análisis posteriormente.

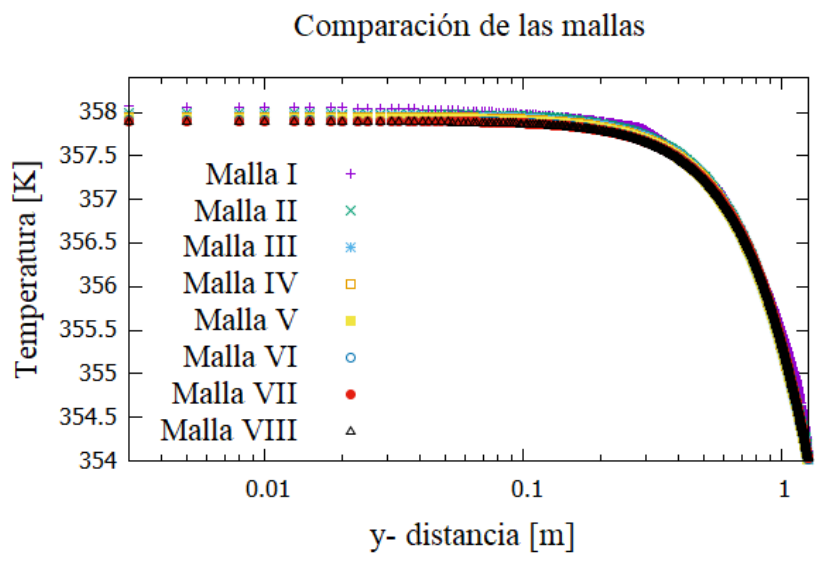

Gráfico 1 Variación de la temperatura a lo largo de la sección transversal para diferentes mallas Fuente: elaboración propia [Gnuplot]

$\begin{array}{rccrc}\text { Malla I } & + & \text { Malla IV } & \text { Malla VII } & \bullet \\ \text { Malla II } & \times & \text { Malla V } & \text { Malla VIII } & \Delta \\ \text { Malla III } & * & \text { Malla VI } & \\ & \text { Comparación de las mallas } & \end{array}$

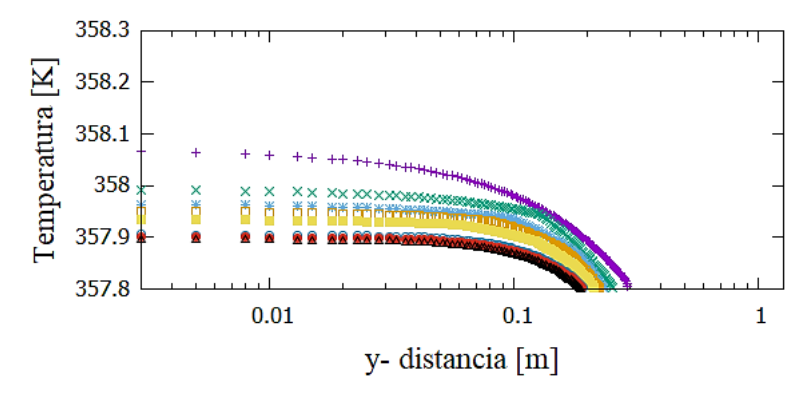

Gráfico 2 Vista a detalle de la variación de la temperatura para las diferentes mallas

Fuente: elaboración propia [Gnuplot]

\begin{tabular}{|r|r|}
\multicolumn{1}{|c|}{ Malla } & \multicolumn{1}{c|}{ Nodos } \\
\hline I & 17400 \\
\hline II & 51200 \\
\hline III & 87000 \\
\hline IV & 177750 \\
\hline V & 590400 \\
\hline VI & 896000 \\
\hline VII & 1208400 \\
\hline VIII & 1274200 \\
\hline
\end{tabular}

Tabla 1 Propiedades termofísicas del agua Fuente: elaboración propia [Word]

La Tabla 1 muestra el número de nodos para cada malla. Se construyen y analizan 8 mallas con diferente número de nodos. La Malla I contiene 17400 nodos y la Malla 8 que es la más densa contiene 1274200 nodos.

ZUÑIGA-CERROBLANCO, José Luis, HORTELANO-CAPETILLO, Juan Gregorio, COLLAZO-BARRIENTOS, Juan Carlos y HERNANDEZ-GUERRERO, Abel. Análisis térmico e hidráulico de diferentes geometrías de tubos para mejorar el desempeño de un radiador de automóvil. Revista de Ingeniería Industrial. 2020 
Como ya se mencionó la malla que se utiliza para realizar el análisis es la Malla VI, se usa esta porque a partir de ella la temperatura encontrada ya no sufre cambios significativos.

La Figura 4 muestra la malla generada para el dominio de la geometría del tubo plano, sobre esta malla se aplicarán las condiciones de frontera y posteriormente se realizará la solución. La malla generada tiene las siguientes características. Nodos: 896,000, Quads=53,014 y Hexas $=708,197$.

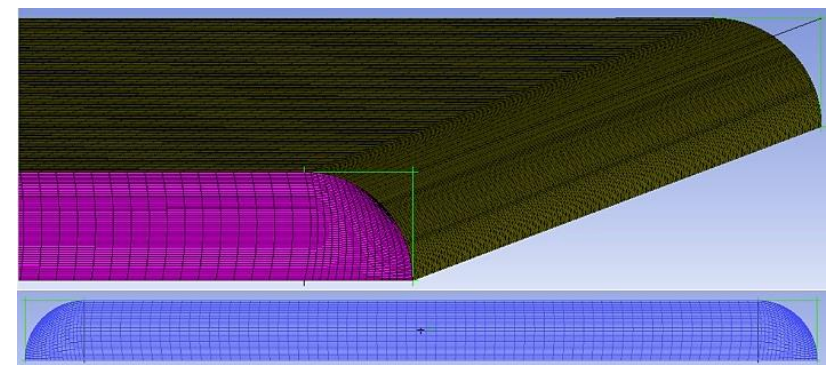

Figura 4 Vista isométrica y frontal de la malla generada para el dominio de la geometría del tubo plano Fuente: elaboración propia [ICEM-ANSYS]

\section{Condiciones de Frontera}

Cuando ya se tienen la malla adecuada se procede a establecer las condiciones de frontera al dominio que se va a analizar. En la Figura 5 se muestran condiciones de frontera asignadas, se tiene una condición de entrada en la superficie por donde entra el fluido que proviene del motor al tubo, una condición de salida en el extremo por donde sale el fluido. En las paredes del tubo se asigna la condición de convección constante. Para no simular todo el tubo y ahorrar tiempo de cómputo se asigna la condición de simetría.

En el análisis numérico se toman ciertas consideraciones que ayudan a simplificar el modelo numérico y a que este logre los criterios de convergencia más rápido. Se toman propiedades constantes para el fluido de enfriamiento, la temperatura del fluido de enfriamiento en la entrada del tubo es de $313 \mathrm{~K}$, el fluido de enfriamiento es una mezcla de $80 \%$ agua y $20 \%$ etilenglicol, la temperatura ambiente se considera de $303 \mathrm{~K}$, el coeficiente convectivo de transferencia de calor en las paredes del tubo es de $50 \mathrm{~W} / \mathrm{m}^{2} \mathrm{~K}$, que es el coeficiente convectivo estimado cuando una automóvil viaja a una velocidad de 70 km/h (Park \& Pak, 2002), estado estable para la transferencia de calor, flujo laminar y el modo de transferencia de calor por radiación es despreciado.

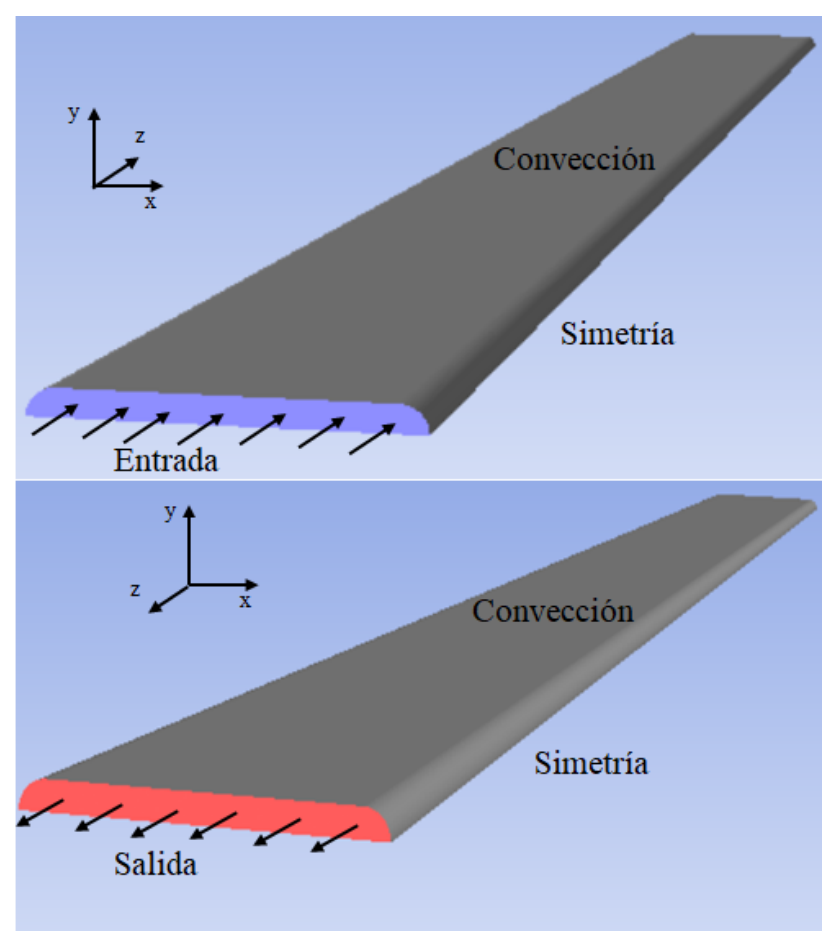

Figura 5 Asignación de las condiciones de frontera Fuente: elaboración propia [ICEM-ANSYS]

\section{Propiedades Termofísicas}

En los sistemas de enfriamiento usados en los automóviles se emplea una mezcla de aguaetilenglicol, el porcentaje de cada uno de estos depende de las condiciones ambiental en las cuales se vaya a usar el automóvil. En el presente trabajo se usa una mezcla de $80 \%$ agua y $20 \%$ etilenglicol. Las propiedades termofísicas como la densidad, la viscosidad, la conductividad térmica y el calor especifico usadas en este análisis son las que se presentan en la Tabla 2. Las propiedades para la mezcla $80 \%$ agua $-20 \%$ etilenglicol son tomadas del trabajo publicado por (Peyghambarzadeh, Hashemabadi, Naraki, \& Vermahmoudi, 2013).

\begin{tabular}{|l|r|r|r|}
\hline \multicolumn{1}{|c|}{$\begin{array}{c}\text { Propiedad } \\
\text { Termofísicas }\end{array}$} & Agua & Etilenglicol & $\begin{array}{c}\text { Agua 80\%- } \\
\text { Etilenglicol } \\
\text { 20\% }\end{array}$ \\
\hline $\begin{array}{l}\text { Densidad } \\
\rho\left[\mathrm{kg} / \mathrm{m}^{3}\right]\end{array}$ & 992 & $1101^{\mathrm{a}}$ & 1008 \\
\hline $\begin{array}{l}\text { Viscosidad } \\
\mu[\mathrm{kg} / \mathrm{m}-\mathrm{s}]\end{array}$ & 0.00065 & $0.0095^{\mathrm{b}}$ & 0.0019 \\
\hline $\begin{array}{l}\text { Conductividad } \\
\text { térmica } \\
k\left[\mathrm{~W} / \mathrm{m}-{ }^{\circ} \mathrm{C}\right]\end{array}$ & 0.633 & $0.256^{\mathrm{b}}$ & 0.58 \\
\hline $\begin{array}{l}\text { Calor } \\
\text { especifico } \\
C_{P}\left[\mathrm{~J} / \mathrm{kg}-{ }^{\circ} \mathrm{C}\right]\end{array}$ & & & 4020 \\
\hline
\end{tabular}

Tabla 2 Propiedades termofísicas, densidad, viscosidad conductividad térmica y calor especifico, del agua pura, etilenglicol puro y una mezcla de agua $80 \%$ y etilenglicol $20 \%$ a $40{ }^{\circ} \mathrm{C}$ 


\section{Ecuaciones Gobernantes}

Las ecuaciones gobernantes que se deben solucionar para el domino que se desea analizar son: ecuación de conservación de masa (ecuación 1), ecuaciones de momento (ecuaciones 2-4) y la ecuación de energía (ecuación 5).

$\frac{\partial u}{\partial x}+\frac{\partial v}{\partial y}+\frac{\partial w}{\partial z}=0$

$\rho\left(u \frac{\partial u}{\partial x}+v \frac{\partial u}{\partial y}+w \frac{\partial u}{\partial z}\right)=-\frac{\partial p}{\partial x}+\mu \nabla^{2} u$

$\rho\left(u \frac{\partial v}{\partial x}+v \frac{\partial v}{\partial y}+w \frac{\partial v}{\partial z}\right)=-\frac{\partial p}{\partial y}+\mu \nabla^{2} v$

$\rho\left(u \frac{\partial w}{\partial x}+v \frac{\partial w}{\partial y}+w \frac{\partial w}{\partial z}\right)=-\frac{\partial p}{\partial z}+\mu \nabla^{2} w$

$\rho c_{\mathrm{p}}\left(u \frac{\partial T}{\partial x}+v \frac{\partial T}{\partial y}+w \frac{\partial T}{\partial z}\right)=k \nabla^{2} T$

\section{Validación del modelo numérico}

Todo modelo numérico requiere ser validado, para comprobar que las consideraciones tomadas, el domino y las ecuaciones gobernantes se resuelven de forma adecuada. Para la validación del modelo numérico, se toma la correlación obtenida por (Vajjha, Das, \& Ray, 2015), la cual obtuvieron para un tubo tipo plano, el fluido de enfriamiento que usaron es etilenglicol puro, para el cual tomaron una temperatura de $40{ }^{\circ} \mathrm{C}$ a la entrada del tubo de enfriamiento. Realizaron experimentos para flujo laminar y turbulento, obteniendo correlaciones para ambos casos. La ecuación (6) muestra la correlación que obtuvieron para flujo laminar, la cual se utilizara para la validación del modelo numérico.

$N u=1.9421\left(\operatorname{RePr} \frac{D_{h}}{z}\right)^{0.3}\left(\operatorname{RePr} \frac{D_{h}}{z}\right) \geq 33.33$

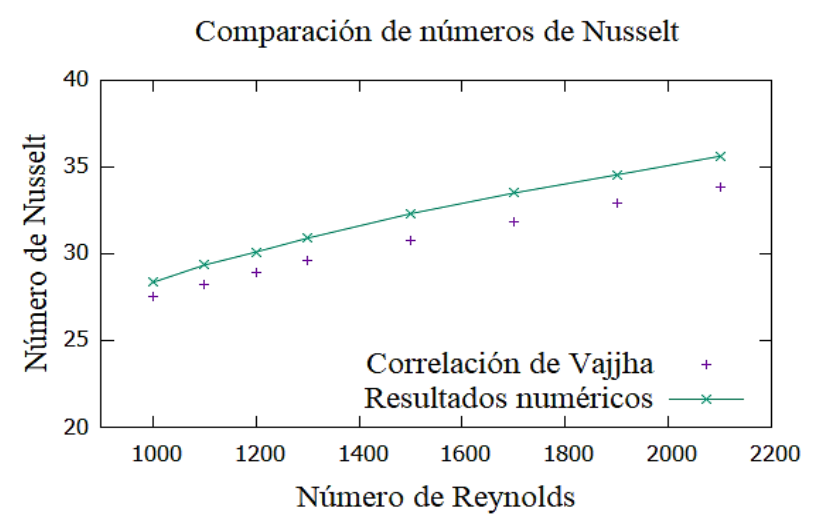

Gráfico 3 Comparación de los resultados numéricos obtenidos para etilenglicol puro con la correlación experimental obtenida por Vajjha

Fuente: elaboración propia [Gnuplot]
En el Gráfico 3 se puede observar la comparación de los resultados numéricos obtenidos con el modelo numérico de este trabajo con los resultados de la correlación obtenida por Vajjha (ecuación 6). Se puede observar que los resultados del modelo numérico tienen una buena concordancia que los resultados calculados con la correlación, lo cual nos indica que el modelo numérico puede utilizarse para realizar el estudio.

Para realizar una comparación entre las diferentes geometrías que se analizan se calcula para cada una de ella la generación de entropía, la cual se utiliza para cuantificar el rendimiento del sistema.

La ecuación (7) determina la generación de entropía local, se compone de dos partes, la primera que determina la generación de entropía local debido a los efectos de la transferencia de calor, para realizar este cálculo se utilizan los campos de temperaturas; una segunda parte de la ecuación determina la generación de entropía local debido a los efectos de la mecánica de los fluidos, en este caso se utilizan los campos de velocidades para realizar el cálculo, con esto se tienen contemplados ambos efectos, los que se producen debido a la transferencia de calor y los producido por la caída de presión.

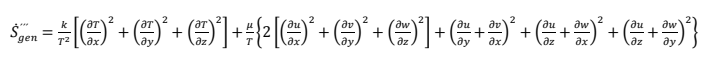

\section{Resultados}

Dentro de los resultados obtenidos se encuentran los de transferencia de calor y los hidrodinámicos.

Para la transferencia de calor se obtiene el número de Nusselt, el Gráfico 4 muestra el número de Nusselt obtenido para diferentes números de Reynolds, esto para el análisis del tubo plano. Se puede observar, como es de esperarse, un incremento del número de Nusselt conforme la velocidad del fluido de enfriamiento se incrementa, obteniendo de esta manera una mayor transferencia de calor para números mayores de Reynolds. 


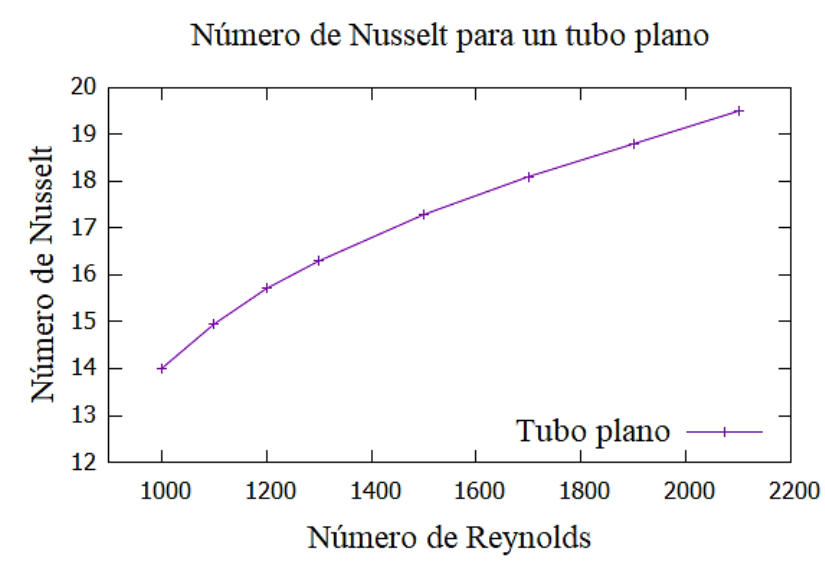

Gráfico 4 Número de Nusselt para diferentes números de Reynolds para la configuración de un tubo plano Fuente: elaboración propia [Gnuplot]

En el Gráfico 5 se muestra la diferencia de presión entre la entrada y la salida del tubo plano. Se observa que conforme aumenta la velocidad del fluido de trabajo, el incremento en la diferencia de presiones aumenta también, requiriendo de esta manera una mayor cantidad de potencia suministrada por la bomba de circulación para mover el fluido de enfriamiento.

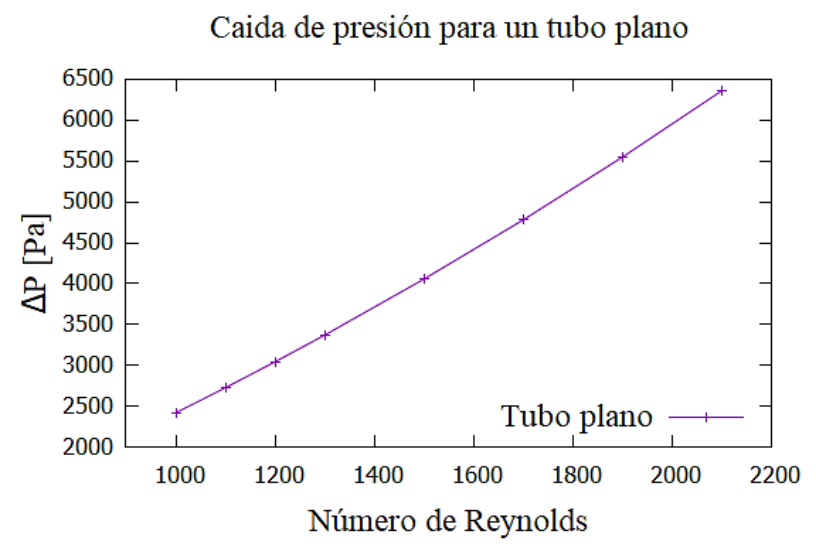

Gráfico 5 Diferencia de presión a lo largo del tubo para diferentes números de Reynolds para la configuración de un tubo plano

Fuente: elaboración propia [Gnuplot]

Una vez obtenidos los números de Nusselt y la caída de presión para cada caso de estudio, se realiza una comparación de las diferentes geometrías utilizadas, para poder hacer una elección de cuál es la que ofrece un mejor rendimiento térmico e hidráulico.

En el Gráfico 6 su muestra una comparación de las diferentes geometrías estudias en este trabajo, se observa que la geometría que presenta el mayor número de Nusselt es la del tubo plano, seguida por el perfil cuadrado y el elíptico.
Esto nos indica que la geometría del tubo plano presenta una mayor remoción de calor proveniente del fluido de enfriamiento hacia el ambiente, donde la cantidad de calor removida proviene directamente del motor.

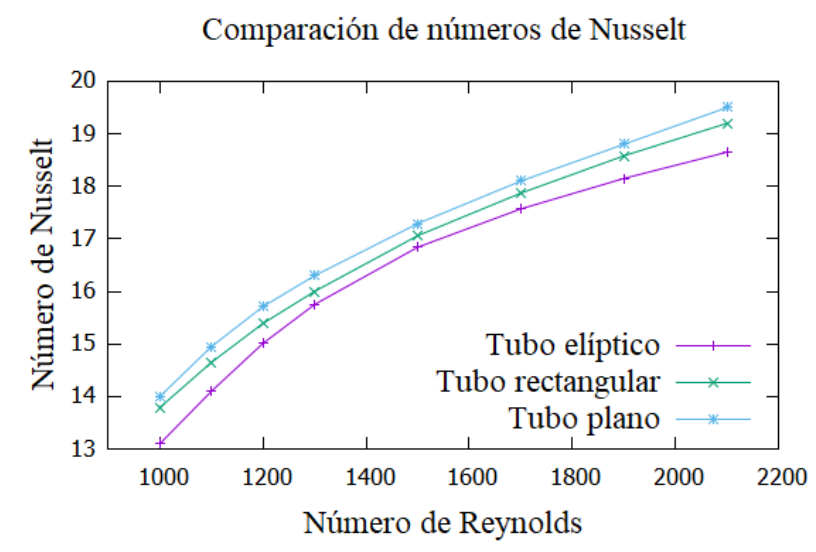

Gráfico 6 Número de Nusselt para diferentes números de Reynolds para la configuración de un tubo plano, rectangular y elíptico

Fuente: elaboración propia [Gnuplot]

Así como se hace una comparación de la transferencia de calor, también se compara la diferencia de presión para cada una de las geometrías. En el Gráfico 7 se puede observar la diferencia de presión para cada una de las geometrías analizadas. Se observa que todas las geometrías presentan el mismo comportamiento, se tiene que la geometría con una menor caída de presión es la elíptica, y la que presenta la mayor caída de presión en la geometría rectangular. Por lo tanto, la geometría que requiere menos energía para mover el fluido de enfriamiento a través de los tubos contenidos en el radiador es la geometría elíptica.

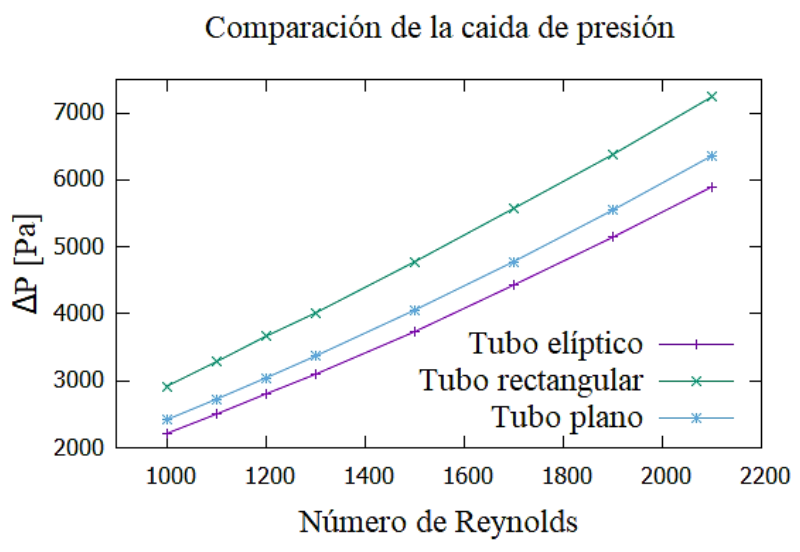

Gráfico 7 Diferencia de presión a lo largo del tubo para diferentes números de Reynolds para la configuración de un tubo plano, rectangular y elíptico

Fuente: elaboración propia [Gnuplot] 
De las gráficas anteriores se obtiene que la geometría que ofrece un mejor rendimiento térmico es la del tubo plano, pero también esta tiene una mayor diferencia de presión que la del tubo elíptico, si el objetivo es tener una mejor de transferencia de calor sin importar la caída de presión, se puede elegir la geometría del tubo plano.

Si por otro lado se requiere una menor caída de presión, se elige la del tubo elíptico. Es por lo que se debe obtener un balance entra la transferencia y la caída de presión obtenidas, para esto se usa el análisis de generación de entropía.

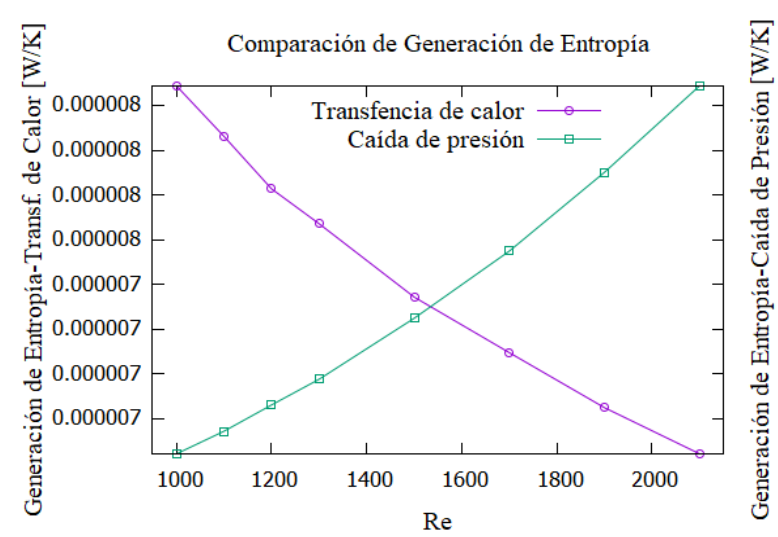

Gráfico 8 Generación de entropía debido a la transferencia de calor y la caída de presión para el tubo plano a diferentes números de Reynolds

Fuente: elaboración propia [Gnuplot]

El Gráfico 8 muestra la generación de entropía debido a la transferencia de calor y a la caída de presión para un tubo plano. Observamos que la entropía generada por la transferencia de calor disminuye con el aumento del número de Reynolds, y que en el caso de la generación de entropía debido a la caída de presión sucede lo contrario, esta aumenta con el aumento del número de Reynols.

Por lo tanto, se debe tener cuidado ya que debe existir un balance entre ambas generaciones de entropía, esto para encontrar un punto óptimo entre la transferencia de calor obtenida y la caída de presión producida.

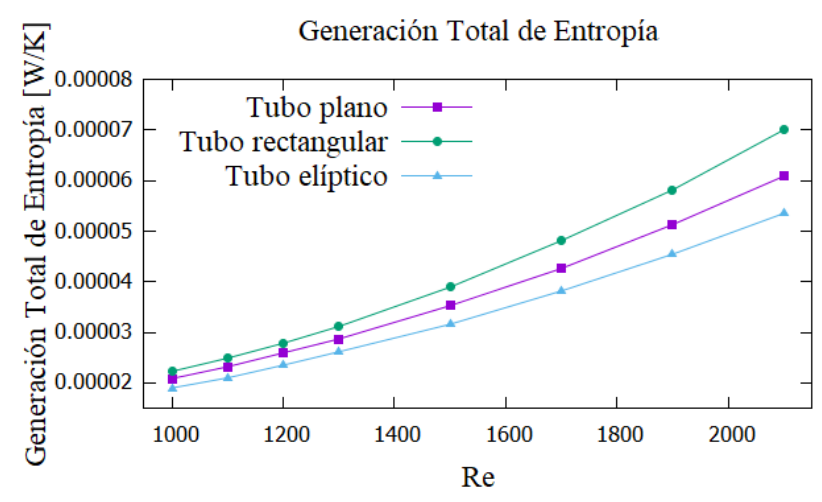

Gráfico 9 Generación de entropía total para las geometrías analizadas en el presente trabajo para diferentes números de Reynolds

Fuente: elaboración propia [Gnuplot]

El Grafico 9 muestra la generación de entropía total para las diferentes geometrías analizadas a diferentes números de Reynolds. Los resultados muestran que la geometría que genera una mayor cantidad de entropía es la cuadrada, la que presenta una menor generación de entropía es la elíptica, es por esto que la geometría elíptica ofrece un mejor rendimiento en cuanto al balance de transferencia de energía y la caía de presión.

\section{Conclusiones}

Se analizan un radiador de calor para el enfriamiento de un automóvil. Diferentes geometrías de la sección transversal de un tubo de radiador de automóvil son estudiadas y comparadas. Se construyen las geometrías en $\mathrm{CAD}$, se malla el domino que se desea estudiar y se realiza un análisis de sensibilidad de malla, para posteriormente obtener la solución de las ecuaciones gobernantes y condiciones de frontera.

Se compara el modelo numérico usado con las correlaciones encontradas en la bibliografía, teniendo que el modelo numérico tiene una muy buena exactitud de cuerdo las correlaciones, esto nos sirve para poder usar el modelo numérico en este y en futuros trabajos.

De los resultados obtenidos se tiene que la geometría del tubo plano ofrece una mayor transferencia de calor, esto debido que los números de Nusselt encontrados son mayores para esta geometría. Por otro lado, la caída de presión para la geometría elíptica es la menor, es decir, esta geometría ofrece la mayor transferencia de calor, pero también se requiere menor energía para mover el fluido de enfriamiento.

ZUÑIGA-CERROBLANCO, José Luis, HORTELANO-CAPETILLO, Juan Gregorio, COLLAZO-BARRIENTOS, Juan Carlos y HERNANDEZ-GUERRERO, Abel. Análisis térmico e hidráulico de diferentes geometrías de tubos para mejorar el desempeño de un radiador de automóvil. Revista de Ingeniería Industrial. 2020 
Considerando solamente la transferencia de calor, la geometría del tubo plano se debería de usar en los tubos que componen un radiador de automóvil, ya que con esto se puede obtener una mayor transferencia de calor.

Por otro lado, se realiza un análisis de generación de entropía, el cual nos indica que la geometría elíptica es la que genera una menor cantidad de entropía, por lo tanto, es la que tiene un mejor balance entre la transferencia de calor y la caída de presión.

En futuros trabajos se propondrá una configuración completa del radiador de calor en base a los resultados aquí obtenidos, esto para comprobar que el radiador cumple con su objetivo de mantener el motor a la temperatura adecuada y obtener una configuración completa del radiador.

\section{Nomenclatura}

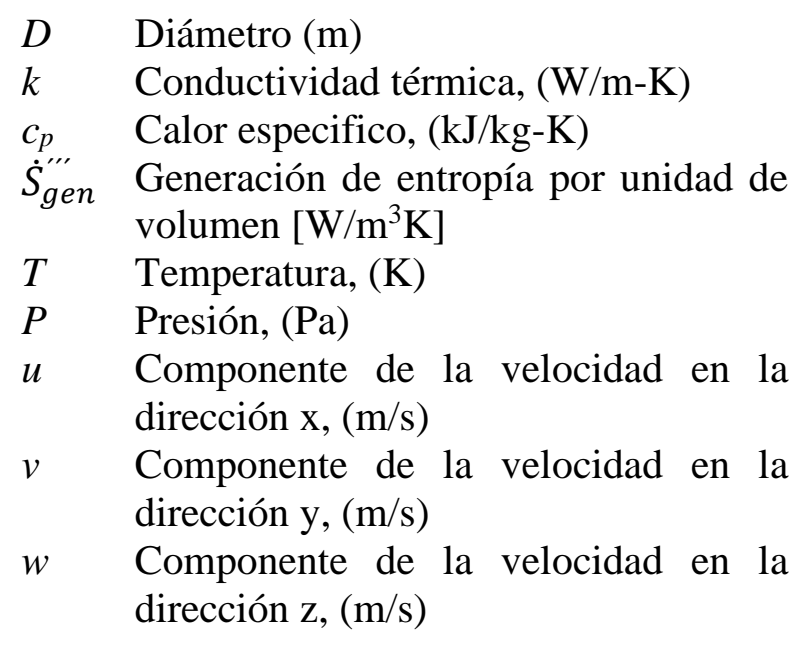

$\begin{array}{ll} & \text { Símbolos griegos } \\ \Delta & \text { Diferencia } \\ \rho & \text { Densidad, }\left(\mathrm{kg} / \mathrm{m}^{3}\right) \\ \mu & \text { Viscosidad dinámica, }(\mathrm{kg} / \mathrm{m}-\mathrm{s})\end{array}$

$\begin{array}{ll} & \text { Subíndice } \\ h \quad \text { Hidráulico }\end{array}$

\section{Referencias}

Achaichia, A., \& Cowell, T. A. (1988). Heat transfer and pressure drop characteristics of flat tube and louvered plate fin surfaces. Experimental Thermal and Fluid Science, 1(2), 147-157.
Agudelo Ortiz, J. V. (2020). Fabricación e instalación de una toma auxiliar de aire para el carenaje del motor de la Aeronave T-90 CALIMA para obtener un mejor rendimiento del sistema de refrigeración.

Avila Navas, H. A. (2020). Modelo didáctico del funcionamiento del motor y tren de potencia, para el taller de automecatronica de la Universidad los Libertadores.

Delavari, V., \& Hashemabadi, S. H. (2014). CFD simulation of heat transfer enhancement of A12O3/water and Al2O3/ethylene glycol nanofluids in a car radiator. Applied Thermal Engineering, 73(1), 380-390.

Dittus, F. W., \& Boelter, L. M. K. (1985). Heat transfer in automobile radiators of the tubular type. International Communications in Heat and Mass Transfer, 12(1), 3-22.

Dong, J., Chen, J., Zhang, W., \& Hu, J. (2010). Experimental and numerical investigation of thermal-hydraulic performance in wavy fin-andflat tube heat exchangers. Applied Thermal Engineering, 30(11-12), 1377-1386.

Farrell, P., Wert, K., \& Webb, R. (1991). Heat transfer and friction characteristics of turbulator radiator tubes. SAE Transactions, 218-230.

Huang, H., Mao, F., \& Zhao, X. (2011). Numerical Simulation of Heat-Transfer Character of Ribbon-Tubular Radiator on Air Side. 2011 International Conference on Computer Distributed Control and Intelligent Environmental Monitoring, 505-508. IEEE.

Huminic, G., \& Huminic, A. (2013). Numerical analysis of laminar flow heat transfer of nanofluids in a flattened tube. International Communications in Heat and Mass Transfer, 44, $52-57$.

Kayastha, K. S. (2015). CFD simulation of heat transfer analysis of automobile radiator using helical tubes. International Journal of Engineering Research and Development, 11(1), 24-35.

Kays, W. M., \& London, A. L. (1984). Compact heat exchangers. 
Park, K. W., \& Pak, H. Y. (2002). Flow and heat transfer characteristics in flat tubes of a radiator. Numerical Heat Transfer: Part A: Applications, 41(1), 19-40.

Patel, H. V, Subhedar, D. G., \& Ramani, B. (2017). Numerical Investigation of performance for Car Radiator Oval Tube. Materials Today: Proceedings, 4(9), 9384-9389.

Peyghambarzadeh, S. M., Hashemabadi, S. H., Naraki, M., \& Vermahmoudi, Y. (2013). Experimental study of overall heat transfer coefficient in the application of dilute nanofluids in the car radiator. Applied Thermal Engineering, 52(1), 8-16.

Shah, R. K., \& London, A. L. (2014). Laminar flow forced convection in ducts: a source book for compact heat exchanger analytical data. Academic press.

Vajjha, R. S., Das, D. K., \& Ray, D. R. (2015). Development of new correlations for the Nusselt number and the friction factor under turbulent flow of nanofluids in flat tubes. International Journal of Heat and Mass Transfer, 80, 353-367. 